\title{
Similarity Laws in Low Speed Switched Reluctance Machines Design
}

\author{
P. Lobato \\ Dep. of Electrical Engineering, \\ ESTSetúbal, Polytechnic \\ Institute of Setúbal, Portugal; \\ pedro.lobato@estsetubal.ips.pt \\ J. A. Dente \\ Instituto Superior Técnico, \\ University of Lisbon, \\ Portugal; \\ edentepc@tecnico.ulisboa.pt
}

\author{
J. F. Martins \\ Dep.of Electrical Engineering, \\ New University of \\ Lisbon/CTS-Uninova, \\ Portugal; \\ jf.martins@fct.unl.pt
}

\author{
A. J. Pires \\ Dep. of Electrical Engineering, \\ ESTSetúbal, Polytechnic \\ Institute of Setúbal/CTS- \\ Uninova Portugal; \\ armando.pires@estsetubal.ips.pt
}

\begin{abstract}
This paper presents a set of similarity laws appropriate for low speed switched reluctance machines design. Design methodologies of switched reluctance machines for low speed applications are generally oriented towards the choice of suitable combinations of stator and rotor poles in regular topologies. The proposed similarity laws provide an easy-to-use and robust methodology to compare different magnetic topologies, regular or non-regular, and have the capability to incorporate thermal and magnetic saturation phenomena by introducing constraints. With the assistance of finite-element analysis the issues surrounding the design of low speed SR multimachines topologies are discussed, leading into consideration of modular non-regular topologies. Using similarity laws, the modular topology is compared with a regular switched reluctance machine. The comparison results point out the reduction of copper losses of the modular topology over the regular topology. This paper extends previous discussions of switched machine design into a more general context.
\end{abstract}

Index Terms - Low Speed Energy Converters, Machine Design, Similarity Laws, Switched Reluctance Generator.

\section{Introduction}

The Switched Reluctance Generator (SRG) research work has been mainly directed at the automotive, aerospace and wind energy domains [1-4]. Previous and older treatments of SRG have focused on the design of high-speed SRG (see [5] and the references cited therein), which mainly rely on the regular magnetic structures [6] of two opposing stator poles, per phase, and a minimal number of rotor poles.

The SR machine can be easily controlled and matched to its load by controlling the instants of energizing and deenergizing the stator phases. Furthermore, aspects as robustness and simple construction (only concentrated coils on the stator), control flexibility, high fault tolerance in a wide speed range, make also this machine suitable for harsh environmental applications [7-8]. The last but not the least, different design techniques, as short flux-path topologies, can be applicable to improve maintenance and machine performance.

For some ten years now, more research efforts have been carried out regarding the use of SRG in direct drive wind energy systems. Though there are different magnetic circuit topologies, SRG design methodologies generally aim to choose the number of stator and rotor poles for regular topologies. The formulation of similarity (or scale) laws proposed in this paper, provides a suitable design tool to compare other SRG topologies, differentiated by diverse characteristics of electric and magnetic circuits, and their relative position. In addition, the use of this scale models methodology makes it easy to incorporate in the study of the machine design other physical phenomena, such as thermal effect and magnetic saturation, by introducing some constraints. Thus, this work underlines dimensional and similarity arguments to extend previous discussions about SRG design into a more general context.

This work is motivated by the application of SRG to directdrive wind turbines and other low speed renewable energy converters. In the context of renewable energy conversion, low speed and high torque machines have gained market space in wind energy applications. With the enlargement of the wind turbine capacity and the trend of exploitation the offshore wind resources makes robustness and reliability vital to the economic operation of wind generators.

Thereby, one of the issues that remains under investigation is the massive, costly, unpredictable yet critical wind turbine gearbox - which thought designed to operate efficiently for 20 years, is beyond doubt, falling far short. Those are the main reasons why some manufacturers and design research teams are pursuing direct drive systems using permanent magnet synchronous generators (PMSG) among other solutions. The option for a SRG study chosen by the authors for the proposed paper, was also supported in the steep rise of NdFeB PM price which is taking the focus off the recently successful directly driven PMSG with tone of NdFeB [9]. Furthermore, the permanent magnets mounted on the rotor need to be carefully protected against thermal and mechanical stresses, which may cause demagnetization and further decrease the PMSG performance.

On the other hand, the SR-machine shows the advantages of simple and robust structure and low cost as there are neither permanent magnets nor winding on the rotor. This paper introduces a simple and already known dimensional similarity-based methodology, built however on another paradigm, ready to be used as a design tool for estimation of SRG characteristics and comparison of SR magnetic structures (topologies). 


\section{SIMILARITY LAWS FORMULATION}

Even though the design flexibility of the switched reluctance machines is consistent with a large number of poles, there are various magnetic topologies which can be candidates to support low speed applications. In spite of that diversity of feasible topologies, there are some intrinsic constraints affecting their dimensions and performance. The differentiation of constraints is considered a priority in adopting a suitable methodology to assist in the selection of the magnetic topology and geometry for a certain application. In this context, a constraint is distinguished by its nature. There are physical and material constraints. Since the operation of these rotating machines is sustained by the magnetic field, the first type of constraints is a result of the physical phenomena involved in the electromagnetic conversion. The second type of constraints emerges from the domain of the material properties, depending on the practical limits of insulators and magnetic materials used. That said, the practical constraint of fixing the flux density, results from the saturation limit inherent to the magnetic material. On the other hand, fixing the temperature variation prevents premature deterioration of insulators and the shortening of the equipment lifetime.

Additionally, variables such as power, losses, weight and temperature, should be taken into account, as they also play an important role in the machine design. The relationships between these variables and the machine dimensions facilitate the estimation of certain machine characteristics. Having in mind a machine design oriented towards a change of scale, models based on similarity laws were chosen.

\section{A. Rated power and losses}

In this type of machines based on magnetic field properties, the frequencies and sizes involved are such, that the displacement-current term in Maxwell's equations can be neglected. Neglecting this term results in the magneto-quasistatic form of Maxwell's equations. In both motoring and generating mode operations, there is a physical constraint due to the electromagnetic linkage of electrical and magnetic circuits. Equation (1) represents this linkage and shows an implicit relationship of characteristic dimensions of each type of circuit.

$$
\text { rot } H \propto J
$$

Considering a change of scale in the construction-oriented machine design, equation (1) can be written in the form of (2), where $\boldsymbol{l}$ represents a characteristic linear dimension.

$$
B \propto J l
$$

This proportional relationship underlines that current excited magnetic circuits impose a scale-factor in their linear dimensions, which is reflected in the relationship $B / J$. Hence, significant changes of performance and machine features can be achieved, by modifying that characteristic linear dimension.

Variables such as power, losses and material weight, are also critical in the machine design. Thus, an attempt is made here to find scale relationships involving copper losses and some of the variables mentioned considering machine dimensions, with the aim of comparing and predicting certain SRG dimensional characteristics and parameters based on similarity laws.

For that purpose, the comprehension of the energy conversion principles of SR machines is a first step, and can be easily described using the generating mode operation represented in Fig.1.

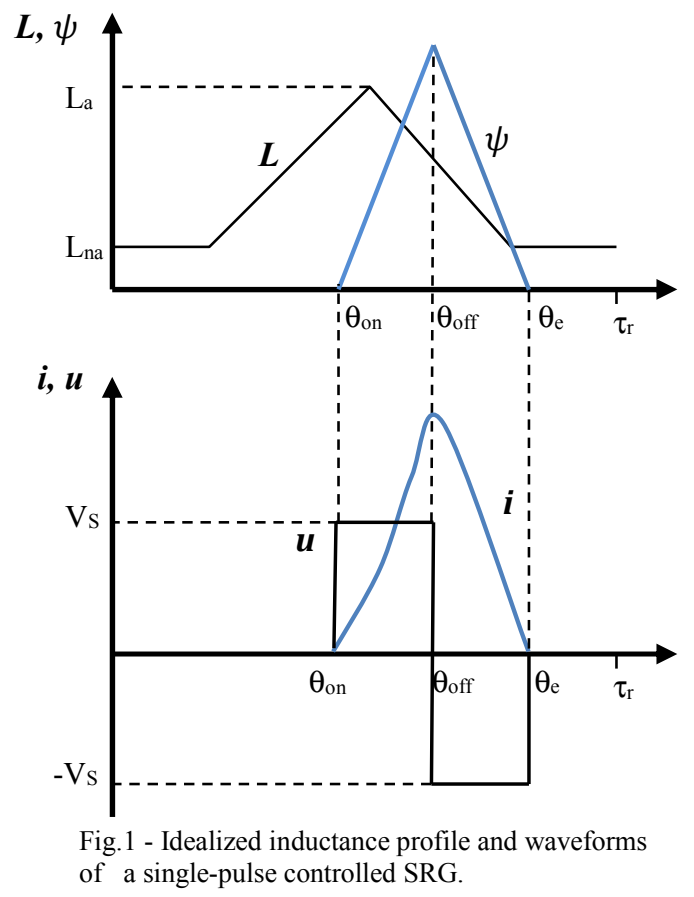

Neglecting saturation phenomena at this point, Fig.1 shows the profiles of idealized inductance $L$, phase flux-linkage $\psi$, phase current $i$ and voltage $\boldsymbol{u}$ for a SRG for single-pulse operation, where $V_{S}$ is the dc-link voltage and $L_{a}, L_{n a}$ are, respectively, the aligned inductance and the unaligned inductance. In a regular SR machine with $N_{R}$ rotor poles, those waveforms are periodic in $\boldsymbol{\tau}_{R}$, that is the rotor polepitch $\left(2 \pi / N_{R}\right)$. During the excitation period, from $\theta_{\text {on }}$ to $\theta_{\text {off }}$, current flows from the supply and energy is stored in the machine magnetic field. After commutation, at $\theta_{\text {off }}$, the excitation energy is returned to the supply and simultaneously, the energy provided by the prime mover is converted to electrical energy.

At $\theta_{e}$, all the flux from excitation period has been extinguished, and no more electrical energy is returned to the supply. If losses are neglected, the output energy over each stroke exceeds the excitation energy by the mechanical energy supplied.

The SR machine is capable of operating continuously as a generator, if the electrical output energy exceeds the 
excitation energy, i.e., by keeping the excitation period so that the bulk of the winding conduction period comes after the aligned position $(d L / d \theta<0)$. At all rotor speeds, it is considered that the SRG can be operated in single pulse mode, which results in low switching losses.

To clarify the issues in exciting the SRG and extracting energy through the phase winding, it is instructive to look at the electrical dynamics of an SRG phase as follows:

$$
u=\frac{d \psi}{d t}+R i
$$

Multiplying both members of eq. (3) with phase current, neglecting the ohmic losses and assuming a non-saturated machine $(\boldsymbol{\psi}=\boldsymbol{L}(\boldsymbol{\theta}) \boldsymbol{i})$, the instantaneous power is given as

$$
u i \cong i \frac{d \psi}{d t} \cong i L \frac{d i}{d t}+i^{2} \frac{d L}{d \theta} \omega
$$

where $\boldsymbol{\omega}$ is the rotor angular speed $(\boldsymbol{\omega}=\boldsymbol{d} \boldsymbol{\theta} / \boldsymbol{d} \boldsymbol{t})$.

The SR energy converter can be represented as a lossless magnetic energy storage system, with electrical and mechanical terminals as shown in Fig. 2. In this type of system, the magnetic field serves as the coupling medium between the electric and mechanical terminals. This sort of representation is valid in situations where the loss mechanism can be separated from the storage mechanism, and is also useful in generating mode operation, where the average torque is negative.

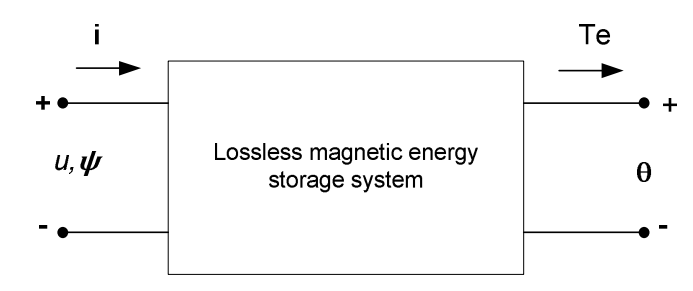

Fig. 2 - Schematic magnetic-field based SR energy converter

For the lossless magnetic energy storage system, the instantaneous power flow in the system can be expressed by

$$
u i=\frac{d W_{m}}{d t}+T_{e} \omega
$$

Where $\boldsymbol{W}_{\boldsymbol{m}}$ is the stored energy in the magnetic field expressed by (6), and $\boldsymbol{T}_{\boldsymbol{e}}$ is the electromagnetic torque for a linear system, the magnetic energy $\boldsymbol{W}_{\boldsymbol{m}}$ and coenergy $\boldsymbol{W}_{\boldsymbol{c}}$ are numerically equal. Thereby, the time rate of change of the magnetic energy is given by (7) and $\boldsymbol{T}_{\boldsymbol{e}}$ can be determined from the coenergy as indicated by (8).

$$
W_{m}=\frac{1}{2} L i^{2}
$$

$$
\begin{gathered}
\frac{d W_{m}}{d t}=i L \frac{d i}{d t}+\frac{1}{2} i^{2} \frac{d L}{d \theta} \omega \\
T_{e}=\frac{\partial W_{c}}{\partial \theta}=\frac{1}{2} i^{2} \frac{d L}{d \theta}
\end{gathered}
$$

After replacing $\boldsymbol{T}_{\boldsymbol{e}}$ in eq. (5), the comparison with eq. (4) allows to conclude that less than half the electrical power involved in the system is converted from the mechanical power of the prime mover. In fact, part of it is being stored in the magnetic field. It should be noted that this argument has been developed for a non-saturated machine and in terms of instantaneous power. However, for scaling design based on similarity laws, average power and torque are considered essential. The average power $\langle\boldsymbol{p}\rangle$ is thus given by (9). Both current and phase inductance may be written as functions of rotor position, and maximum values $\boldsymbol{I}$ and $\boldsymbol{L}_{\boldsymbol{a}}$, respectively, by using (10) and (11). After multiplying these functions, the phase flux-linkage is expressed by (12). The average power per phase is thus given by (13), in such way that the integral expressed therein results in a constant value.

$$
\begin{gathered}
\langle p\rangle=\langle u i\rangle=\left\langle T_{e}\right\rangle \omega \\
i=I \cdot \xi_{(\theta)} \\
L=L_{a} \cdot \chi_{(\theta)} \\
\psi=L i=L_{a} I \cdot \xi_{(\theta)} \chi_{(\theta)} \\
\langle p\rangle=\frac{\omega}{2 \tau_{r}} \int_{0}^{\tau_{r}} i^{2} \frac{d L}{d \theta} d \theta=\frac{\omega}{2 \tau_{R}} I^{2} L_{a} \int_{0}^{\tau_{r}} \xi_{(\theta)}^{2} \frac{d \chi_{(\theta)}}{d \theta} d \theta
\end{gathered}
$$

In terms of dimensional analysis, similarity laws for $\boldsymbol{\psi}, \boldsymbol{I}$ and $\boldsymbol{L}_{\boldsymbol{a}}$ can be written as (14), (15) and (16), by introducing the scale factor $\boldsymbol{l}$. Taking into account (13), one may write a proportional relationship for $\langle\boldsymbol{p}\rangle$ in the form of (17).

$$
\begin{gathered}
\psi \propto B l^{2} \\
I \propto J l^{2} \\
L_{a} \propto \frac{\psi}{I} \propto \frac{B}{J} \\
\langle p\rangle \propto N_{R} \omega\left(J l^{2}\right)^{2} \frac{B}{J} \propto N_{R} \omega B J l^{4}
\end{gathered}
$$

In terms of similarity laws for a m-phase SR machine the rated power is finally expressed by 


$$
P \propto m N_{R} \omega J B l^{4}
$$

Identical similarity laws can be written for motor mode since the physical fundamental concepts are similar. It should also be emphasized that the criteria of selecting a greater or smaller number of variables to be explicit in the similarity laws, depends only on the characteristics and parameters considered relevant for the proposed comparison of topologies. For instance, the number of rotor poles is a significant parameter in evaluating low speed SRG designs, since $B$ is limited by magnetic saturation of the iron, and $J$ should be kept under certain limits due to copper losses and the preservation of the insulating material. For this dimensional analysis, the influence of the sequential activation and deactivation of the phases on the torque was neglected. In fact, by assuming similar levels of B (similar levels of saturation), it is assured that the aforementioned comparison of topologies, in relative terms, is valid. Therefore, a compromise involving the number of poles, dimensions related with both the magnetic and electrical circuits, and phase current is required.

\section{B. Multi-machine topology}

In regard to SR machines design, there are a multiplicity of feasible topologies, differentiated by the properties of the electrical and magnetic circuits, and their relative location. A feasible SRG topology for an energy converter can comprise a series of $\mathrm{n}-\mathrm{SR}$ machines assembled on a common axis. Comparing with a monolithic SR machine, that mono-axial multi-machine topology shows a higher fault tolerance and a simpler maintenance. The previous relationships are suitable to observe some effects and certain limitations of scale laws, when applied in SRG design. In order to apply the methodology, it is considered a SRG characterized by a rated power, copper and iron losses, maximum flux and current densities, temperature and efficiency. These values are assumed as reference values, allowing the comparison with similar machines, but built in a different scale.

In this scale models methodology, applied to an electromagnetic device, there is one degree of freedom to select the variables $B$ and $J$ as both are interrelated by (2).

Although the flux density is significantly different in diverse regions and instants, the saturation levels should be kept constant in all regions of the magnetic circuit. Therefore, the practical limitation imposed by the saturation of magnetic materials means that $J$ can be expressed by $B / l$.

According to similarity laws, it is possible to compare the relative losses of a hypothetical SRG topology, composed of a set of $\boldsymbol{n}$ machines assembled in the same shaft, with a similar monolithic SRG, even though much larger but with the same rated power of the former one. Assuming a constant rotor speed and equal number of phases and rotor poles of both topologies, the scale law for rated power $\boldsymbol{P}_{\boldsymbol{N}}$ can be rewritten simply by

$$
P_{N} \propto B^{2} l^{3}
$$

For one SR module of the multi-machine topology, the rated power can be expressed by

$$
P_{m} \propto B_{m}^{2} l_{m}^{3} \propto B^{2} l_{m}^{3}
$$

where $\boldsymbol{B}$ and $\boldsymbol{B}_{\boldsymbol{m}}$ are the flux densities, considered equal as both topologies use the same core material, and $\boldsymbol{l}_{\boldsymbol{m}}$ is a linear dimension of multi-modular topology. However, this multimodular topology is characterized by the total rated power shared by $\boldsymbol{n}$ SR modules as following:

$$
P_{m}=\frac{P_{N}}{n}
$$

Moreover, for comparison purposes of topologies, one searches a relation that involves linear dimensions of both topologies and number of modules. Using (20) and (21) a scale law can be expressed by

$$
\frac{l_{m}}{l} \propto\left(\frac{1}{n}\right)^{1 / 3}
$$

This result states, as expected, that the higher the number of modules, the lesser is the relative linear dimension. Using the previous proportionality law, one can establish a relationship that allows the comparison of relative copper losses in both topologies as follows:

$$
\frac{P_{J m_{T}}}{P_{J}} \propto \frac{n m J_{m}{ }^{2} l_{m}{ }^{3}}{m J^{2} l^{3}} \propto n\left(\frac{l_{m}}{l}\right) \propto n^{2 / 3}
$$

Under the constant flux density condition, using (22) and (23), the scale law concerning the relative copper losses can be expressed by (24) as represented in Fig. 3.

$$
p_{J r} \propto \frac{P_{J m_{T}}}{P_{N}} \propto n^{2 / 3} P_{N}^{-2 / 3}
$$

In terms of copper losses, it is possible to verify a scale gain for larger monolithic SR machines, rather than a modular system composed by SR units. However, that multi-machine topology could be elected for certain applications where fault tolerance, low cost and easy maintenance are priorities.

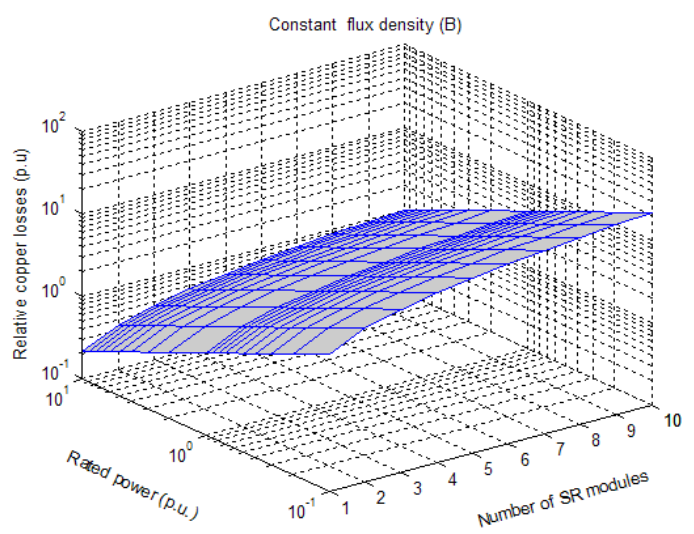

Fig. 3 - Relative copper losses for scale models of SR machines considering magnetic saturation constraint. 


\section{DESIGN STUDY RESULTS AND DISCUSSION}

At this point, and according to the scale criteria, the previous evaluation of the copper losses clearly supports the preference for a monolithic topology, over a multi-machine topology. The selection of a magnetic structure, that takes into account a shorter flux-path, may reduce the magnetomotive force (MMF) absorbed in the iron core.

A long flux-path of the regular SRG is illustrated in Fig.4A, and an example of a short flux-path is shown in Fig.4-B, highlighting the flux closing between two adjacent rotor poles. In order to compare the flux-path length $\boldsymbol{l}_{F s f}$ of a shortflux path (SFP) topology with the length of the regular machine $\boldsymbol{l}_{\boldsymbol{F}}$, an exterior radius $\boldsymbol{R}_{\mathbf{2}}$, similar to both topologies, is assumed. For simplicity of analysis, the circular arc length of the shaft contour is neglected. Using the scale laws, the effect on copper losses can be evaluated in relative terms. The relationship of the flux paths is approached by (25).

$$
\frac{l_{F s f}}{l_{F}} \cong \frac{\frac{\pi}{m}\left(R_{1}+R_{2}\right)+2\left(R_{2}-R_{1}\right)}{\pi R_{2}+2 R_{2}}
$$
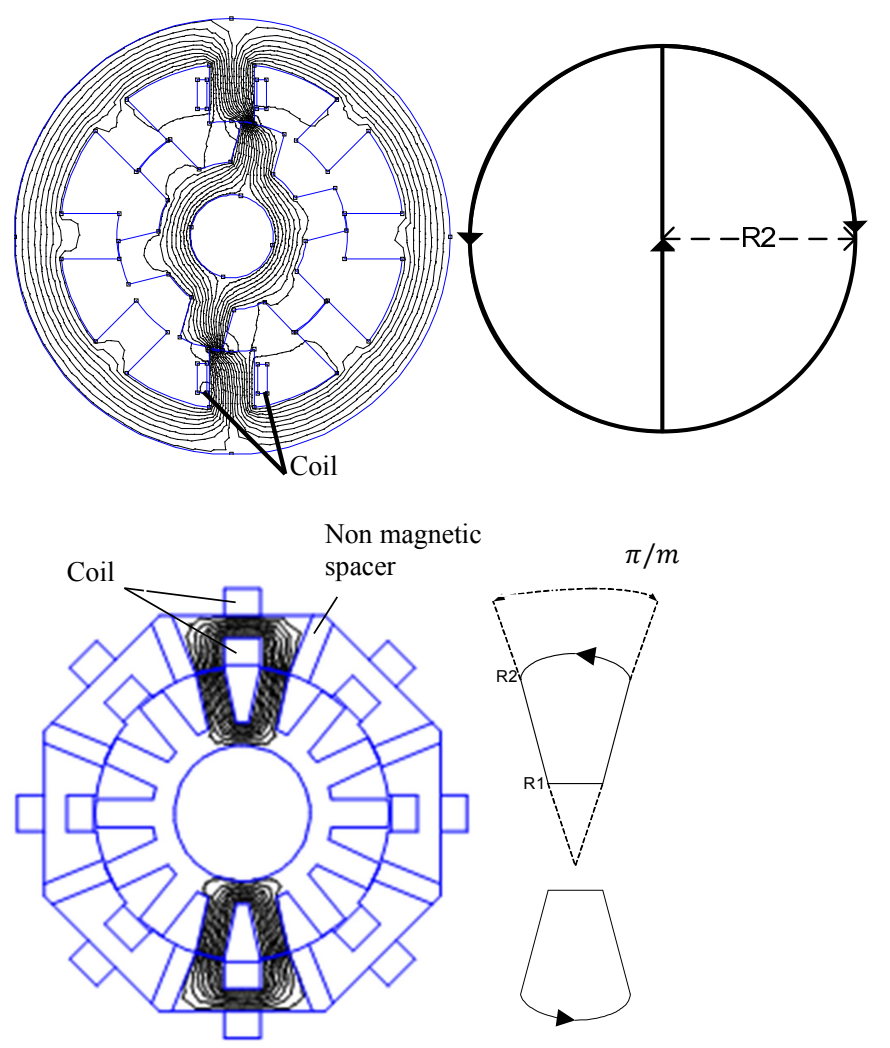

Fig. 4 - A. Long flux-path for a regular topology $\left(m=4 ; N_{R}=6\right)$; B. Short flux-path for a SPF topology $\left(\mathrm{m}=4 ; \mathrm{N}_{\mathrm{R}}=14\right)$ with stator modules separated by non-magnetic spacers.

As inferred from the geometry of both topologies, in the short flux-path case it is considered a relationship between the exterior and interior radius $R_{1}$ given by $R_{2}=3 R_{l}$. Both are four phase machines $\left(m^{\prime}=m=4\right)$, and the SFP topology has a higher number of rotor poles compared with the reference machine, $N_{R}^{\prime}=14$. Taking into account the above assumptions, the ratio of flux-path lengths $l_{F s f} / l_{F}$ is approximately $1 / 2$.

The magnetic structure that was chosen is a modular SFP topology, constituted by eight stator modules separated by non-magnetic spacers. Part of the coil wound on the base of each module is exposed, making the copper cooling process and the heat removal more effective. High fault tolerance, easy maintenance, and simplicity of the manufacturing, are also favorable arguments to choose this SFP topology [10].

In order to improve the use of the conductive material and the core iron, it is of particular interest to introduce a modification into the linear scales. This option is accomplished through a structural change, in which two specific dimensions of each material will be used, $l_{C u}$ e $l_{F}$. The laws related to differentiated scales for copper and iron allow to track the course of the copper losses of the SFP topology, with respect to the regular 8/6 topology. In such case, the copper characteristic dimension plays a key role in limiting the temperature increase which can be expressed by (26).

$$
J \propto l_{C u}{ }^{1 / 2}
$$

A limited increase of temperature and a constant flux density, yields the relationship between the iron and copper characteristic dimensions expressed by (27) and the copper losses by (28).

$$
\begin{gathered}
\mathrm{Bl}_{\mathrm{F}} \propto \mathrm{l}_{\mathrm{Cu}}{ }^{3 / 2} \\
P_{J} \propto m J^{2} l_{C u}{ }^{3} \propto m l_{C u}{ }^{2} \propto m\left(B l_{F}\right)^{4 / 3}
\end{gathered}
$$

The copper losses depend upon the flux path length becoming lower in the SFP topology. The decrease of copper losses at a wide power range suggests a rescaling operation, i.e. a reduction of the dimensions of the SFP machine with respect to the regular machine, considering identical rated power.

A finite-element analysis is used to evaluate some starting hypothesis of this scale methodology. Fig.5-B shows that the mutual flux-linkage is very small when compared with the flux-linkage of each phase. Therefore, in a similar way to regular topologies, the mutual flux-linkage between two phase windings of the SFP magnetic topology is negligible. As shown in Fig. 5-A, the magnetic saturation has a significant effect in this modular SFP topology. However, a steep saturation can also be seen in regular machines as presented in [11]. Two structurally different topologies (for instance, having different numbers of poles) can be compared, preserving certain dimensions of the magnetic circuit where the flux paths lie on. Thus, the stator exterior diameter, the air gap length, the radius of the air gap as well as the core length, should be fixed and kept constant. 

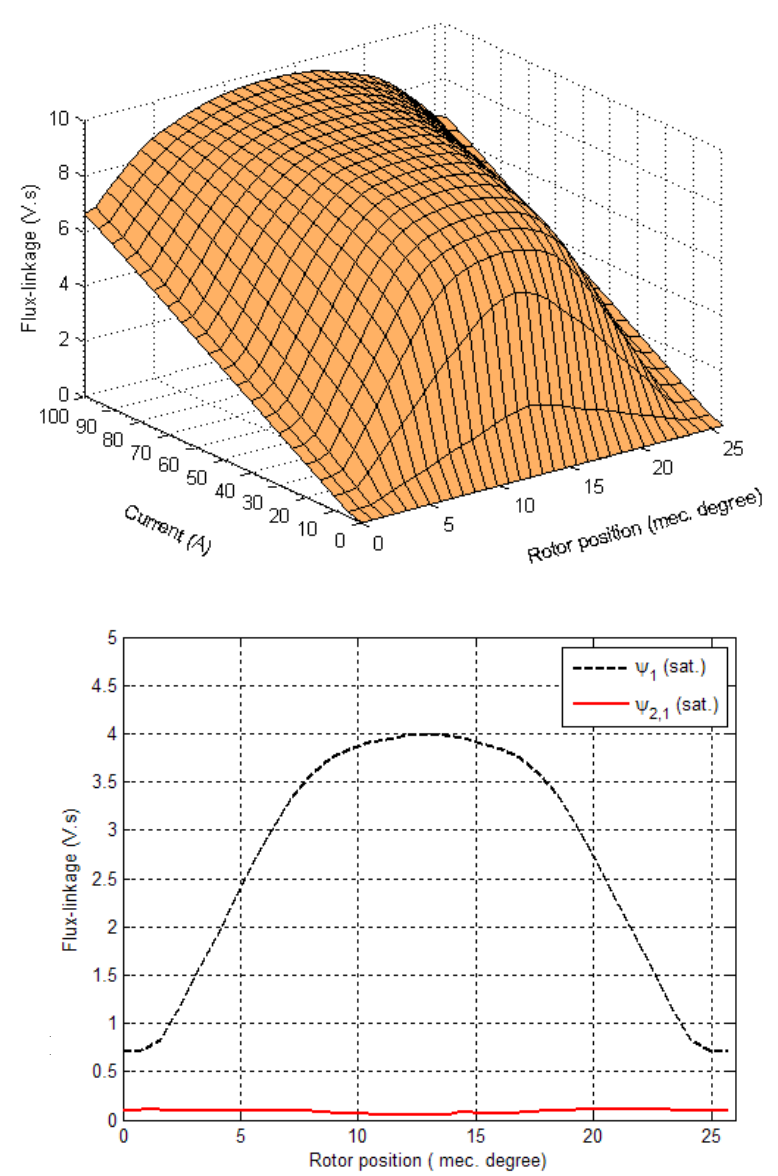

\section{CONCLUSIONS}

A comparative analysis based on similarity laws has been presented for low speed SR Machines. The comparison and evaluation of magnetic structures plays an important role in the SR machines design. Besides covering the evaluation of stator/rotor poles combinations for regular switched reluctance machines, the formulation of scale laws proposed is also suitable to compare other SR topologies, distinguished by different characteristics of electric and magnetic circuits and their own relative location. As shown by the authors, this methodology includes other physical phenomena that take place during machine operation, like thermal changes and magnetic saturation, by introducing some constraints.

This work highlights the usefulness and effectiveness of the similarity laws formulation, as an assistant tool for SR machine design. Furthermore, its application to regular and non-regular SR topologies clearly emphasizes some design details of magnetic structures in machine performance. Ultimately, in the field of direct drive energy converters and other low speed renewable energy systems, this scale methodology helps the designer to achieve a SRG design with a minimum of trial-and-error sizing, when comparing different feasible SRG topologies. As a guideline, and not as an end, the comparison results achieved in this dimensional analysis indicate that additional work should be developed concerning a detailed design of the modular SFP topology. Considering real dimensions and material characteristics, a full-scale machine design will make it possible to compare initial costs of the modular SFP SRG topology with classic generators using gearbox.

\section{ACKNOWLEDGEMENTS}

This work was supported by national funds through IPS Polytechnic Institute of Setúbal and FCT - Fundação para a Ciência e a Tecnologia, CTS - Centre of Technology and Systems, under project UID/EEA/00066/2013.

\section{REFERENCES}

[1] B. Bilgin, A. Emadi, and M. Krishnamurthy, "Design considerations for switched reluctance machines with a higher number of rotor poles", IEEE Trans. Industrial Electronics, vol.59, pp. 3745-3756, Oct. 2012.

[2] B. Fahimi, A.R.B. Emadi and Jr. Sepe, "A switched reluctance machine based starter/alternator for more electric cars", IEEE Trans. Energy Conversion, vol. 19, pp. 116-124, March 2004.

[3] Y.J. Bao, K.W.E. Cheng, N.C. Cheung, and S.L. Ho , "Experimental examination on a new switched reluctance wind power generator system for electric vehicles", IET Power Electronics, vol. 5, pp. 1262-1269, Sept. 2012.

[4] H. Yahia, N. Liouane, and R. Dhifaoui, "Differential evolution methodbased output power optimisation of switched reluctance generator for wind turbine applications", IET Renewable Power Generation, vol. 8, pp.795-806, Sept. 2014.

[5] P. Chancharoensook, and M.F. Rahman, "Control of a four-phase switched reluctance generator: experimental investigations", in Proc. IEEE International Electric Machines and Drives Conference, vol.2, pp.842-848, June 2003.

[6] T.J.E Miller, Switched Reluctance Motors and Their Control, Magna Physics Publishing and Clarendon Press, 1993.

[7] I. Kioskeridis, C. Mademlis., "Optimal efficiency control of switched reluctance generators", IEEE Trans. Power Electronics, vol. 21, pp.1062 - 1071, July 2006.

[8] R. Cardenas, R. Pena , M. Perez, J. Clare, G. Asher and P. Wheeler, "Control of a switched reluctance generator for variable-speed wind energy applications", IEEE Trans. Energy Conversion, vol. 20, pp.781 791, 2005.

[9] I. Boldea, L. Tutelea, F. Blaabjerg, "High power wind generator designs with less or no PMs: an overview", in Proceedings 17th International Conference on Electrical Machines and Systems (ICEMS), Hangzhou, China, Oct. 2014.

[10] M. Ruba, I. Viorel, and L. Szabó, "Modular stator switched reluctance motor for fault tolerant drive systems", IET Electric Power Applications, vol. 7, pp.159-169, March 2013.

[11] B. Parreira, S. Rafael, A. J. Pires, and P. J. Costa Branco, "Obtaining the magnetic characteristics of an 8/6 switched reluctance machine: FEM analysis and experimental tests", IEEE Trans. Industrial Electronics, vol.52, pp.1635-1643, Dec. 2005. 